\title{
Fragmentation of Percolation Clusters at the Percolation Threshold
}

\author{
Mark F. Gyure ${ }^{(a)}$ and Boyd F. Edwards \\ Department of Physics, West Virginia University, Morgantown, West Virginia 26506
}

(Received 11 March 1991; revised manuscript received 6 January 1992)

\begin{abstract}
A scaling theory and simulation results are presented for fragmentation of percolation clusters by random bond dilution. At the percolation threshold, scaling forms describe the average number of fragmenting bonds and the distribution of cluster masses produced by fragmentation. A relationship between the scaling exponents and standard percolation exponents is verified in one dimension, on the Bethe lattice, and for Monte Carlo simulations on a square lattice. These results further describe the structure of percolation clusters and provide kernels relevant to rate equations for fragmentation.
\end{abstract}

PACS numbers: $82.20 .-\mathrm{w}, 05.40 .+\mathrm{j}, 05.70 . \mathrm{Jk}$

Physical processes such as polymer degradation, collisions, combustion, and grinding cause particles to break into smaller particles. Recent interest in the fragmentation of random porous solids [1,2] provides motivation for examining the fragmentation characteristics of simple models of these materials. Perhaps the simplest model of a spatially random solid is the bond percolation cluster, and the simplest method of breaking such a cluster is by removing a bond chosen at random. The consequences of such fragmentation are intimately tied to the structure of these clusters. This Letter describes new information about the structure of percolation clusters obtained by examining their fragmentation characteristics.

The structure of percolation clusters has received considerable attention in recent years because of the desire to understand transport properties through random media. For this reason, much of the recent effort has focused on the structure of clusters at and near the percolation threshold $p_{c}$ where the infinite cluster dominates the behavior of the system. In particular, the link, node, and blob picture of the infinite cluster has helped to clarify the conduction of electricity through random networks and the flow of water through random porous materials $[3,4]$. Since the backbone of the infinite cluster carries current across the cluster, the structure and connectivity of the backbone has been examined carefully [5-7]. Comparatively little effort has been spent, however, on characterizing overall cluster connectivity, an important issue for fragmentation.

In this spirit, we ask about the consequences of removing a bond of mass 1 from a percolation cluster of finite mass $s$, given by the number of bonds, at the percolation threshold $p_{c}$. Is the cluster easily broken or is it more likely to remain connected? Also, if it does break, what are the relative masses $s^{\prime}$ and $s-s^{\prime}-1$ of the resulting "daughter" clusters? Are clusters more likely to break into daughters of comparable or of vastly different masses? To uniquely quantify these questions, we define an ensemble average number $a_{s}$ of "fragmenting" bonds on a cluster of mass $s$ and a probability $b_{s}$ ' of obtaining a daughter cluster of mass $s^{\prime}$ by fragmentation of a cluster of mass $s$. For the bond cluster of mass $s=11$ in Fig. 1, removing any of three fragmenting bonds (thin lines) breaks the cluster, with $s^{\prime}=2$ for fragmenting bond 1 . Bond clusters are particularly simple because, in contrast with site clusters, fragmentation of a bond cluster of any dimensionality is "binary," always producing two daughters. The functions $a_{s}$ and $b_{s}$ 's are clearly fundamental to understanding the fragmentation of percolation clusters; their determination is the primary goal of this Letter.

Rate equations [8-11] describe the time evolution of the mass distribution of a system of particles subject to fragmentation. Solutions of such equations depend on prior knowledge of the particle breakup rate and the distribution of daughter masses upon fragmentation. Although these "kernels" contain the essential physics of the particle morphology and of the fragmentation process, their mass dependences are typically assumed out of mathematical convenience. For random bond dilution of percolation clusters, the breakup rate is proportional to $a_{s}$ and the distribution of daughter masses is just $b_{s^{\prime} s}$. Thus, calculation of $a_{s}$ and $b_{s} s$ supplies essential physics for a rate-equation approach to percolation.

The key to understanding the fragmentation of a percolation cluster lies in determining which bonds are singly connected in the sense that their removal causes the cluster to break into two distinct parts. These fragmenting bonds are closely related to "red" bonds on the cluster backbone [4,5]. The backbone is the set of bonds through which current flows when two bonds designated $i$ and $j$ are maintained at different potentials. Red bonds are

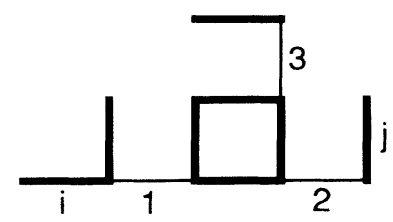

FIG. 1. Example of an eleven-bond cluster on a square bond lattice. There are three fragmenting bonds on this cluster (thin lines) and two of these ( 1 and 2) are red bonds on the backbone between bonds $i$ and $j$. 
singly connected bonds on the backbone whose removal results in a loss of connectivity (and current) between $i$ and $j$. In Fig. 1, fragmenting bonds 1 and 2 serve as red bonds with $i$ and $j$ as shown, whereas bond 3 does not. The set of red bonds for a cluster is not unique; designation of different $i$ and $j$ on the same cluster results in a new backbone and a new set of red bonds. The identification of a fragmenting bond on a finite cluster does not depend, however, on the designation of two reference bonds; the set of fragmenting bonds on a cluster is unique. The scaling properties of red bonds have been studied because they are essential to understanding the connectivity across the incipient infinite cluster. In the same way that the study of red bonds on the backbone of the incipient infinite cluster is fundamental to understanding the conductivity of random networks, the study of fragmenting bonds on finite clusters is fundamental to understanding the way random materials break up.

Explicit scaling forms for $a_{s}$ and $b_{s}$ ' can be found for bond clusters in one dimension (linear chains) and on the Bethe lattice. For $d=1$, all but the two end bonds of a chain of length $s$ are fragmenting bonds and all daughter masses are equally likely, so that [10] $a_{s}=s-2$ and $b_{s^{\prime} s}=2 /(s-2)$. For the Bethe lattice, $a_{s}=\left(1-B_{s 0}\right) s$ and $b_{s^{\prime} s}=\left(s / a_{s}\right) B_{s s^{\prime}}$ follow from [12] $B_{s s^{\prime}}$, the probability of obtaining a cluster of mass $s^{\prime}$ by removing an arbitrary bond (not restricted to fragmenting bonds as for $b_{s^{\prime}}$ ) from a cluster of mass $s$. For both $d=1$ and the Bethe lattice [13], these $p$-independent functions obey the scaling forms

$$
\begin{aligned}
& a_{s} \sim s^{\lambda}, \\
& b_{s^{\prime} s}=s^{-} g\left(s^{\prime} / s\right)
\end{aligned}
$$

for large clusters, with $\lambda=\phi=1$ for linear chains and $\lambda=1, \phi=\frac{3}{2}$, and $g(x) \sim x^{-3 / 2}(1-x)^{-3 / 2}$ for the Bethe lattice. Thus, for the Bethe lattice, the daughter distribution scales as the daughter/parent mass ratio $x=s^{\prime} / s$ and fragmentation is less likely to produce daughters of comparable masses than of vastly different masses.

For finite $d>1$ the situation is considerably more complicated due to the $p$ dependence of cluster structure [14]; fragmentation properties are likewise expected to be $p$ dependent. At $p_{c}$, however, the scaling forms given by Eqs. (1) are expected to apply with, perhaps, different exponents. In the absence of exact solutions for finite $d>1$, we propose Eqs. (1) as scaling forms for fragmentation of clusters at $p_{c}$ and perform Monte Carlo simulations on a square lattice to test this proposal for $d=2$.

The simulations for $d=2$ employ clusters generated at $p_{c}$ on a $1200 \times 1200$ square lattice using a Leath algorithm. Finite-size effects are negligible [15] for clusters of mass less than 50000 grown on this lattice. To determine whether a bond on a cluster of mass $s$ is a fragmenting bond, it is removed and one of its ends is selected as a starting point. The mass of the subcluster connected to that point is then determined by a burning algorithm. If the mass of the subcluster is zero or $s-1$ or if the other end of the bond is reached during the burning process, then the bond is nonfragmenting. Otherwise, the bond is fragmenting and the mass of the subcluster is the mass $s$ of one of the daughters.

The computed average number of fragmenting bonds per cluster as a function of the cluster mass $s$ (Fig. 2), though consistent with the large-s scaling form $a_{s} \sim s^{\lambda}$, indicates that corrections to scaling are important. A detailed correction-to-scaling analysis of data from 66024 clusters yields a computed value [13] $\lambda=1.001 \pm 0.006$ for $d=2$ which includes the value $\lambda=1$ obtained for $d=1$ and for the Bethe lattice.

To estimate $\phi$, we define an ensemble average number $c_{s^{\prime} s}=a_{s} b_{s^{\prime} s}$ of daughters of mass $s^{\prime}$ on a cluster of mass $s$ and compute its first moment $\mu_{s}^{(1)}=\sum_{s^{\prime}} s^{\prime} c_{s^{\prime} s} \sim s^{\psi}$ involving a sum over the smaller daughters $s^{\prime}$ and a combined exponent $\psi=2+\lambda-\phi$. A linear fit to the corresponding data in Fig. 2, obtained from 39109 clusters and satisfying $s \geq 3162$, where corrections to scaling are negligible [13], yields $\psi=1.400 \pm 0.006$. The computed value $\phi=1.601 \pm 0.008$ then follows from the computed value of $\lambda$. A correction-to-scaling analysis [13] yields a value $\psi=1.396 \pm 0.007$ and a comparable value $\phi=1.605$ \pm 0.009 .

A plot of the computed scaled daughter distribution $s^{\phi} b_{s} s$ using the value $\phi=1.601$ versus the scaled daughter mass $s^{\prime} / s$ (Fig. 3) shows striking agreement with the proposed form $b_{s^{\prime} s}=s^{-} g\left(s^{\prime} / s\right)$. The computed scaling function $g\left(s^{\prime} / s\right)$ for $d=2$ has the same basic features as the exact scaling function for the Bethe lattice, including

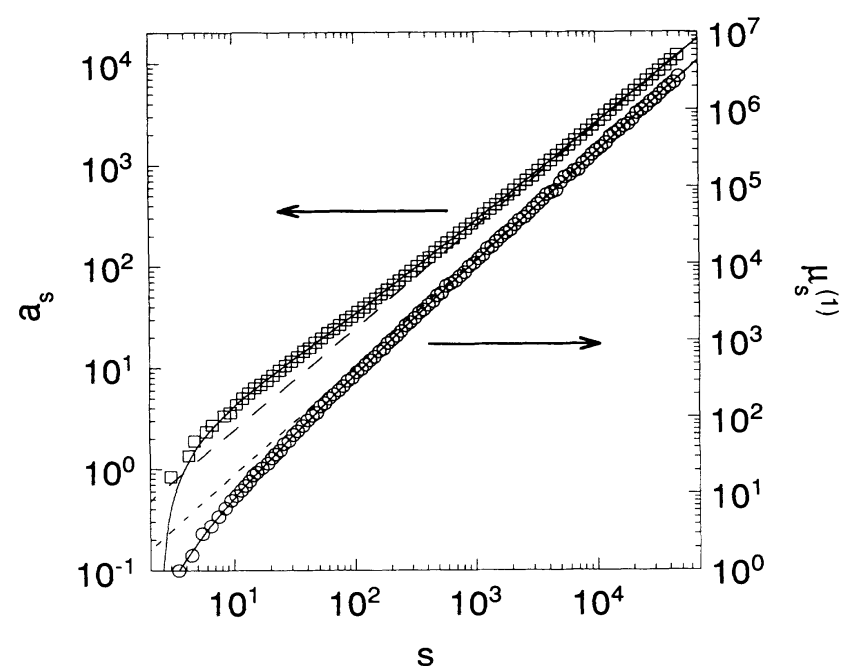

FIG. 2. Computed average number of fragmenting bonds $a_{s} \sim s^{\lambda}$ (squares) and first moment $\mu_{s}^{(1)}=\sum_{s^{\prime}} s^{\prime} c_{s^{\prime} s} \sim s^{2+\lambda-\bullet}$ (circles) of the number $c_{s}$ s of daughters of mass $s^{\prime}$ on clusters of mass $s$ on a square bond lattice at $p_{c}$. Dashed lines are powerlaw fits with $\lambda=1.001$ and $\phi=1.601$. Solid traces include correction-to-scaling terms. 


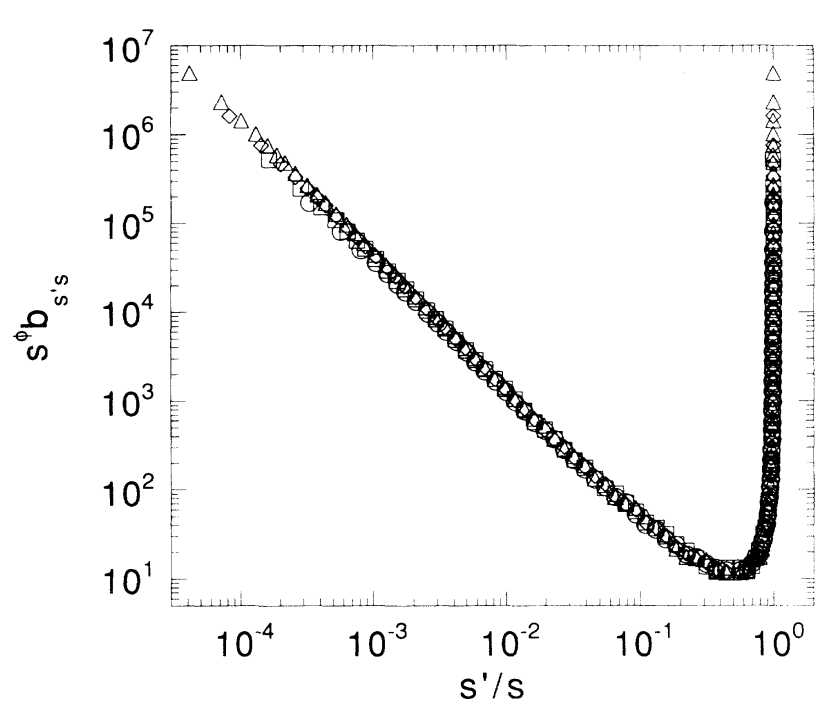

FIG. 3. Computed scaled daughter distribution $s^{\star} b_{s^{\prime} s}$ at $p_{c}$ as a function of the scaled daughter mass $s^{\prime} / s$ plotted for $\phi=1.601$ and for parent masses $s$ in the ranges 4096-4467 (circles), 8192-8933 (squares), 16 384-17867 (diamonds), and 3276835734 (triangles). Slight deviations from scaling are evident for small daughter masses $s^{\prime}$ (leftmost points for each range of $s)$.

symmetry about $x=s^{\prime} / s=1 / 2$ guaranteed for binary fragmentation.

To relate $\lambda$ and $\phi$ to standard percolation exponents, we now develop a scaling theory involving the number $\Lambda_{i j}$ of red bonds between two bonds $i$ and $j$ on a $d$-dimensional lattice. Clearly, $\Lambda_{i j}=0$ if $i$ and $j$ are on the same blob or are not on the same cluster. For $i$ and $j$ in Fig. $1, \Lambda_{i j}=2$. It is helpful to consider the sum $\sum_{i \text { on } l, j} \Lambda_{i j}$ over all bonds $j$ on a single realization of a finite-size lattice of linear dimension $L$ and over bonds $i$ on a particular cluster $l$, noting that only bonds $j$ on that cluster contribute to the sum. The number of times a fragmenting bond $k$ is counted in the sum equals the number of distinct combinations of $i$ and $j$ on the cluster for which bond $k$ is counted as a red bond. This number is just twice the product $s_{k l}^{\prime}\left(s_{l}-s_{k l}^{\prime}-1\right)$ of the masses of the daughters produced by removing the bond, where $s_{k l}^{\prime}$ is the mass of the smaller daughter and $s_{l}$ is the mass of cluster $l$. An exact relationship between the backbone and fragmentation properties of a single cluster follows by summing over all fragmenting bonds on the cluster:

$$
\begin{aligned}
\sum_{i \text { onl }, j} \Lambda_{i j} & =2 \sum_{k} s_{k l}^{\prime}\left(s_{l}-s_{k l}^{\prime}-1\right) \\
& =\sum_{s^{\prime}} C_{s^{\prime}}^{(l)} s^{\prime}\left(s_{l}-s^{\prime}-1\right) .
\end{aligned}
$$

The convenient second equality involves a sum over all daughter masses $s^{\prime}$ produced by fragmentation of the cluster, where

$$
C_{s^{\prime}}^{(l)}=\sum_{k}\left(\delta_{s^{\prime}, s_{k l}^{\prime}}+\delta_{s^{\prime}, s_{l}-s_{k l}^{\prime}-1}\right)
$$

is the number of daughters of mass $s^{\prime}$ on the cluster. Equation (2) is easily verified for the cluster in Fig. 1, for which $\sum_{i \text { on } l, j} \Lambda_{i j}=68$.

The next step is to sum Eq. (2) over all clusters $l$ on the lattice, which yields the exact result

$$
\sum_{i j} \Lambda_{i j}=\sum_{s} \sum_{s^{\prime}} C_{s^{\prime} s} s^{\prime}\left(s-s^{\prime}-1\right) .
$$

The sum over $i$ and $j$ on the left-hand side is unrestricted because $\Lambda_{i j}=0$ when $i$ is on an unoccupied bond. The right-hand side involves a sum over cluster masses $s$ and a total number $C_{s^{\prime} s}=\Sigma_{l} \delta_{s, s_{l}} C_{s^{\prime}}^{(l)}$ of daughters of mass $s^{\prime}$ on clusters of mass $s$ on the lattice. The corresponding ensemble average,

$$
\sum_{i j}\left\langle\Lambda_{i j}\right\rangle \sum_{s} \sum_{s^{\prime}} c_{s^{\prime} s}(L) N_{s}(L) s^{\prime}\left(s-s^{\prime}-1\right),
$$

over all realizations of the lattice involves the average number $c_{s^{\prime} s}(L)=\left\langle C_{s^{\prime} s}\right\rangle / N_{s}(L)$ of daughters of mass $s^{\prime}$ per cluster of mass $s$ on a finite lattice of linear dimension $L$, where $N_{s}(L)$ is the total number of clusters of mass $s$ on such a lattice.

Finite-size scaling forms allow us to evaluate Eq. (4). The scaling form $N_{s}(L)=L^{d} n_{s}(L)$ is the product of the number $L^{d}$ of bonds on the $d$-dimensional lattice and the number [14] $n_{s}(L)=s^{-\tau} f\left(s / L^{d_{f}}\right)$ of clusters of mass $s$ per lattice bond at $p_{c}$, where $d_{f}$ is the fractal dimension of percolation clusters at $p_{c}$. The quantity $c_{s^{\prime} s}(L)$ is the finite-lattice version of $c_{s^{\prime} s}=a_{s} b_{s^{\prime} s} \sim s^{\lambda-\phi_{g}}\left(s^{\prime} / s\right)$. Clusters of mass $s$ comparable to the largest typical cluster mass $L^{d_{f}}$ that will fit on the finite lattice are more compact and have fewer fragmenting bonds than typical clusters of mass $s$ on the infinite lattice. Accordingly, the appropriate scaling form $c_{s^{\prime} s}(L) \sim s^{\lambda-\phi} f_{2}\left(s^{\prime} / s, s / L^{d_{f}}\right)$ for the number of daughters must cut off with increasing $s$ near $L^{d_{f}}$; this cutoff behavior is verified numerically in Ref. [13]. To complete the necessary scaling forms, we recall the average number [4] $\left\langle\Lambda_{i j}\right\rangle=r_{i j}^{2-d-\eta+1 / v} h\left(r_{i j} / L\right)$ of red bonds between bonds $i$ and $j$ on a finite lattice, where $r_{i j}$ is the Euclidian distance between these bonds. Substituting these forms yields the scaling behavior $L^{2+d-\eta+1 / v}$ and $L^{d+d_{f}\left(4+\lambda-\phi_{-}-\tau\right)}$ of the left- and right-hand sides of Eq. (4), respectively.

Finally, use of the standard scaling relationships $2-\eta=\gamma / v$ (Ref. [16]), $v d_{f}=\sigma^{-1}$, and $\gamma=(3-\tau) / \sigma$ (Ref. [14]) results in the desired scaling relationship

$$
\psi=1+\sigma
$$

involving the combined exponent $\psi=2+\lambda-\phi$ and the cluster-number scaling exponent $\sigma$. This relationship holds exactly for $d=1$ where $\sigma=1$ and for the Bethe lattice where $\sigma=\frac{1}{2}$ (Ref. [14]). For $d=2$, our linear-fit result $\psi=1.400 \pm 0.006$ includes the exponent [14] $1+\sigma$ $=1+\frac{36}{91} \approx 1.396$. These confirmations give compelling evidence for the validity of $\mathrm{Eq}$. (5).

Results for $d=1, d=2$ (on the square lattice), and the Bethe lattice indicate the possibility that $\lambda=1$ for all $d$. 
This would imply that the fraction $a_{s} / s$ of fragmenting bonds on large clusters at $p_{c}$ is a specific finite number equal to $1,0.24 \pm 0.02$, and $1-[(z-2) /(z-1)]^{z-1}$, respectively, for $d=1, d=2$, and the $z$-coordinated Bethe lattice $(z \geq 3)$. This is consistent with the notion that large clusters at $p_{c}$ are ramified and are easily broken, whereas $\lambda<1$ would imply a vanishingly small fraction of fragmenting bonds and $\lambda>1$ would imply an unphysical fraction greater than unity. Furthermore, the small fraction for $d=2$ reflects the importance of loops in that dimension [4]. Using the likely value $\lambda=1$ in $\psi=2+\lambda$ $-\phi=1.400 \pm 0.006$ (from the linear fit) instead of the computed value of $\lambda$ implies a higher-precision estimate $\phi=1.600 \pm 0.006$ for $d=2$.

The scaling theory and simulations presented here are restricted to binary fragmentation on bond lattices. It would be interesting to study nonbinary fragmentation on site lattices to determine whether $\phi$ is universal and to explore the symmetries of the scaling functions. Exploration of the $p$ dependence of $a_{s}$ and $b_{s}$, particularly the $p=0$ limit, should be interesting and could be useful in the context of rate-equation approaches to percolation.

We are grateful to Martin Ferer for numerous insightful discussions on scaling theory. We also gratefully acknowledge stimulating discussions with Paul Beale, Mark Bradley, Greg Huber, Alan Kerstein, Sidney Redner, Eugene Stanley, and Robert Ziff. This work was supported by Department of Energy Grant No. DE-FG22-89PC89791, National Science Foundation Grant No. RII8922106, and the West Virginia University Energy and
Water Research Center.

(a) Present address: Center for Polymer Studies, Department of Physics, Boston University, Boston, MA 02215.

[1] S. Redner, in Statistical Models for the Fracture of Disordered Media, edited by H. J. Herrmann and S. Roux (Elsevier, New York, 1990), Chap. 10, and references therein.

[2] M. Sahimi, Phys. Rev. A 43, 5367 (1991); M. Sahimi, G. R. Gavalas, and T. T. Tsotsis, Chem. Eng. Sci. 45, 1443 (1990), and references therein.

[3] H. E. Stanley, J. Phys. A 10, L211 (1977).

[4] A. Coniglio, J. Phys. A 15, 3829 (1982).

[5] R. Pike and H. E. Stanley, J. Phys. A 14, L169 (1981).

[6] H. J. Herrmann and H. E. Stanley, Phys. Rev. Lett. 53, 1121 (1984).

[7] T. Ohtsuki and T. Keyes, J. Phys. A 17, L267 (1984).

[8] E. D. McGrady and R. M. Ziff, Phys. Rev. Lett. 58, 892 (1987).

[9] Z. Cheng and S. Redner, J. Phys. A 23, 1233 (1990); Phys. Rev. Lett. 60, 2450 (1988).

[10] M. Cai, B. F. Edwards, and H. Han, Phys. Rev. A 43, 656 (1991); B. F. Edwards, M. Cai, and H. Han, Phys. Rev. A 41, 5755 (1990).

[11] J. Huang, B. F. Edwards, and A. D. Levine, J. Phys. A 24, 3967 (1991).

[12] A. R. Kerstein, J. Phys. A 22, 3371 (1989).

[13] B. F. Edwards, M. F. Gyure, and M. V. Ferer (to be published).

[14] D. Stauffer, Introduction to Percolation Theory (Taylor and Francis, London, 1985).

[15] P. L. Leath, Phys. Rev. B 14, 5046 (1976).

[16] J. W. Essam, Rep. Prog. Phys. 43, 833 (1980). 